\title{
Anaerobic oxidation of methane does not attenuate thermokarst lakes methane emissions
}

\author{
NOAM LOTEM ${ }^{1}$, DR. ANDRE PELLERIN ${ }^{1}$, KATEY \\ WALTER-ANTHONY ${ }^{2}$ AND ORIT SIVAN ${ }^{1}$ \\ ${ }^{1}$ Ben Gurion University of the Negev \\ ${ }^{2}$ Unviersity of Alaska Fairbanks \\ Presenting Author: noamlot@gmail.com
}

The ongoing global temperature rise induces the thawing of permafrost soils in the arctic, allowing Pleistocene-aged frozen organic matter to become available for microbial degradation and production of greenhouse gases. The methane in particular, can play a key role in a positive climate feedback loop, as its global warming potential is $\sim 28$ times larger than the equivalent carbon dioxide. Our study focuses on anaerobic oxidation of methane $(\mathrm{AOM})$ in the sediments of thermokarst lakes, which are the rapidly expanding lakes forming as a result of thawing permafrost. Several cores were collected from the sediments of 4 thermokarst lakes in Alaska in two seasons. Since methane concentrations in the porewater were above saturation, methane production and consumption rates were quantified by incubation experiments. Our results demonstrate that while methane production is vigorous in these sediments, methane oxidation is lower by two orders of magnitude. In the deep sediments (50-90 $\mathrm{cm}$ ), AOM rates were $<1 \%$ of the methane production rate, while in shallow sediments AOM rates were $1 \%-5 \%$ of methane production rates. Experimental evidence indicates that the mechanism of AOM is strongly related to the methane production mechanism, as the addition of methanogens inhibitor prevented AOM, and the ratio of AOM to methane production was not affected by changes in temperature or lithology. Furthermore, electron acceptor additions did not stimulate AOM. This might suggest that the observed AOM signals are the result of enzymatic reversibility ('back-flux') during methane production, rather than thermodynamically favorable AOM. Regardless of the AOM mechanism, our quantitative results show that the thermokarst sediments in this study have little to no buffer-mechanism capable of attenuating methane production. This observation is probably the case in most arctic landscapes, because of the similar geochemical conditions in most interior thermokarst lakes. 